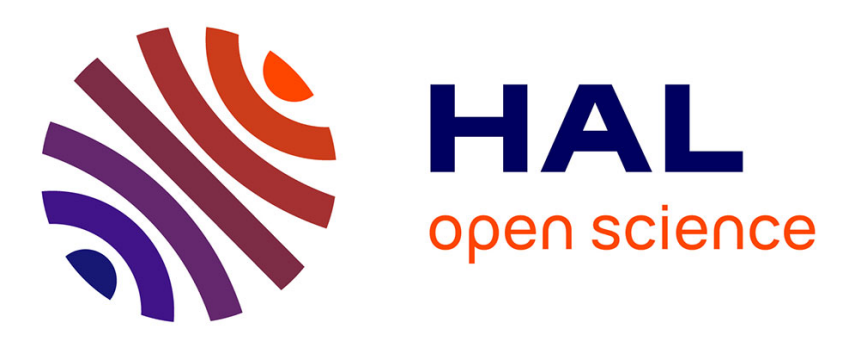

\title{
Conformal representation of Kerr space-time poloidal sub-manifolds
}

Loïc Chantry, Véronique Cayatte, Christophe Sauty

\section{To cite this version:}

Loïc Chantry, Véronique Cayatte, Christophe Sauty. Conformal representation of Kerr space-time poloidal sub-manifolds. Classical and Quantum Gravity, 2020, 37 (10), pp.105003. 10.1088/13616382/ab8399 . hal-03035891

\section{HAL Id: hal-03035891 \\ https://hal.science/hal-03035891}

Submitted on 3 Dec 2020

HAL is a multi-disciplinary open access archive for the deposit and dissemination of scientific research documents, whether they are published or not. The documents may come from teaching and research institutions in France or abroad, or from public or private research centers.
L'archive ouverte pluridisciplinaire HAL, est destinée au dépôt et à la diffusion de documents scientifiques de niveau recherche, publiés ou non, émanant des établissements d'enseignement et de recherche français ou étrangers, des laboratoires publics ou privés. 


\title{
Conformal representation of Kerr space-time poloidal sub-manifolds
}

\author{
Loïc Chantry, Véronique Cayatte, Christophe Sauty \\ LUTH, Observatoire de Paris, UMR 8102 CNRS/INSU, Université Paris Diderot, 5 \\ place Jules Janssen, 92190 Meudon, France \\ LUPM, Université de Montpellier, UMR 5299 CNRS/IN2P3, cc072, place Eugène \\ Bataillon, 34090 Montpellier, France \\ E-mail: loic.chantry@obspm.fr
}

17 March 2020

\begin{abstract}
We use two conformal transformations to represent field lines in the poloidal sub-manifold of Kerr space-time. The first one is based on an embedding in $\mathbb{R}^{3}$ of a manifold which is conform to the poloidal submanifold. The second one is a planar representation using quasi-isotropic coordinates.

We compare plots of the poloidal magnetic field lines in the usual Boyer-Lindquist Cartesian coordinates) and in the conformal representation based on quasi-isotropic coordinates. In a conformal representation these lines appear entering in the horizon perpendicularly to it as deduced through a mathematical perspective conversely to the usual physical approach.

We also compare the value of the conformal factor in these two representations.
\end{abstract}

\section{Introduction}

Generally, stationary and axi-symmetric magnetic field lines penetrate the horizon of a rotating black hole horizon perpendicularly. Indeed, the line element appearing in the expression of the magnetic field $B^{\hat{\theta}}$ latitudinal component reaches a singular value on the horizon. Let call $A$ the magnetic flux across a surface, which border is a circle of constant colatitude and constant Boyer-Lindquist radius. If $A$ is of class $\mathcal{C}^{1}$ across the horizon, we have $B^{\hat{\theta}}=0$ on this horizon. More details on this point are given after Eq. 19. Nevertheless, when the field is not a purely radial field (more precisely if we do not have $\partial_{r} A=0$ on the horizon), this property does not appear clearly in the ordinary field line representation of the poloidal magnetic field. In the literature focusing on black hole magnetosphere, Nathanail and Contopoulos [2014]; Pan [2018] or Mahlmann et al. [2018], the field line representation of some solutions of the Grad-Shafranov equation (GSE) seems not enter in the horizon perpendicularly to it. This problem does not lie with the solution determination. However, this is a typical problem encountered in the planar representation of field lines on non-Euclidean geometry. 
To avoid this kind of problem in the representation, a solution consists in getting isometric embedding in $\mathbb{R}^{3}$ of the 2-dimensional poloidal sub-manifolds on which we represent field lines. In this case, the representation of lines on the isometric embedding should respect angles and lengths. Embedding of two dimensional sub-manifolds of space time gives us in addition to a representation of these manifolds a correct representation of the curves, the lengths and the angles on these surfaces. That is why quickly after the discovery of Kerr-Newman space-time, Smarr [1973] exhibited an embedding of its horizon. This embedding is possible only for an angular momentum below a given value. Hledík et al. [2008] found the isometric embedding of the equatorial plane for Schwarzschild, Ernst or Kerr-Newman space-times. Han and Lee [2016] constructed embedded diagrams of the equatorial surface of a slightly deformed Kerr space-time. We also find embedding of ergosphere surfaces in Ray et al. [2015]. The embedding is not a problem if one of the coordinates is ignorable, i. e. if there is a symmetry in the problem. For the poloidal manifold of the Kerr Black Hole, this is unfortunately not the case. The general case is still an object of research. There is a lot of literature about the construction of embedding algorithms for Riemanian manifolds with positive curvature everywhere, e.g. Bondarescu et al. [2002]; Jasiulek and Korzyński [2012]; Ray et al. [2015] and Tichy et al. [2014].

Nevertheless, since the poloidal manifold of Kerr space time has no ignorable coordinate, its immersion is more complex. The purpose of this paper is to find a correct representation of angles even if the distances are not be correctly represented. We want to explore different ways for tracing curves such that they give a correct measure of angles. Thus, even if we do not obtain a correct representation of distances, we will have a correct representation of angles. Correct representation of angles is important when we represent magneto-hydrodynamics (MHD) fields where cross and scalar products are present in the mathematical formulation of Euler equation (in the Lorentz force for example). The problem of conformal representation was encountered by the navigators of the $\mathrm{XVI}^{t h}$ century. At this time, it was crucial to build a planar representation of the Earth, which properly respects angles between the curves. One famous example is the famous cylindrical map construction proposed by the geographer Mercator.

About the poloidal manifold, we note that a choice of a correct set for conformal factors allows us to go back to the situation with latitudinal symmetry for the conformal manifold. Then we are able to obtain an isometric embedding in $\mathbb{R}^{3}$ for this set of conformal manifold. We present one of these embeddings.

Remembering that any manifold is conformally flat, we can find in our set the one that is embedded in the plane. The radial coordinate of this plane corresponds to the so called quasi-isotropic coordinate. It is used in general relativity, especially for a stationary, axisymmetric and circular space-time Gourgoulhon [2010].

Then we illustrate the conformal properties of these two conformal representations. First, we build and represent two orthogonal families of curves. Then we propose different representations of typical field lines used for astrophysical jets around compact 
objects. We will focus on how the field lines look close to the horizon. Finally, we discuss the differences between the conformal factor values of these two conformal representations.

The conformal representation of poloidal manifolds might be useful for any work requiring a representation of phenomena in the poloidal manifold of a Kerr black hole. This could be useful for representation of electro-dynamics or MHD fields, but also for trajectories of test-mass objects or photons in this plane. The same kind of work can be done for the equatorial sub-manifold of Kerr space-time.

Our results are also valid for Kerr-Newman poloidal sub-manifold replacing the dimensionless spin $a$ by $\sqrt{Q^{2}+a^{2}}$ where $Q^{2}=Q^{2} / 4 \pi \epsilon_{0} \mathcal{G M}^{2}$, with $Q$ the electrical charge of the black hole, the only difference being the value of the conformal factor.

\section{The Kerr poloidal sub-manifold}

Let note $(\mathcal{K}, \mathbf{g})$ the Kerr space time, and $(t, r, \theta, \phi)$ the Boyer-Lindquist coordinates, which map this manifold. In these coordinates let us remind that the metrics may be written as,

$$
d s^{2}=-h^{2} c^{2} d t^{2}+h_{r}^{2} d r^{2}+h_{\theta}^{2} d \theta^{2}+h_{\phi}^{2}\left(d \phi-\beta^{\phi} c d t\right)^{2},
$$

where the introduced functions depend on $(r, \theta)$ and the two parameters, which characterize a Kerr black hole, are its angular momentum $\mathcal{J}$ and its mass $\mathcal{M}$.

Let us introduce the $\mathbf{2 D}$ poloidal sub-manifold of the $4 \mathrm{D}$ Kerr manifold. This submanifold is obtained by $\mathcal{P}_{t, \phi}=\left\{M \in \mathcal{K} \mid r(M) \geq r_{\mathcal{H}} t(M)=t\right.$ and $\left.\phi(M)=\phi\right\}$ taking the part of the Kerr space-time outside of the horizon with constant time and toroidal coordinates. The Kerr poloidal sub-manifold can be mapped with $(r, \theta)$ and its induced metrics is,

$$
d \sigma^{2}=\frac{r^{2}+a^{2} \cos ^{2} \theta}{r^{2}+a^{2}-2 r_{g} r} d r^{2}+\left(r^{2}+a^{2} \cos ^{2} \theta\right) d \theta^{2},
$$

where $2 r_{g}=r_{s}=2 \mathcal{G M} / c^{2}$ is the gravitational radius linked to black hole mass $M$, and $a=\frac{\mathcal{J}}{M c}$ the typical length associated to the angular momentum $\mathcal{J}$. We also have $r_{\mathcal{H}}=r_{g}\left(1+\sqrt{1-\left(a / r_{g}\right)^{2}}\right)$ for the horizon radius. Because of stationarity and axisymmetry of the Kerr space-time manifold, for any value of $t, \phi$, the submanifold $\mathcal{P}_{t, \phi}$ has the same metrics line elements. As a consequence, it also has the same intrinsic geometry. Since the metrics of $\mathcal{P}_{t, \phi}$ is independent of $t, \phi$, we note $\mathcal{P}$ the poloidal sub-manifold.

The dimensionless radial coordinate is $\tilde{r}=r / r_{g}$ and the dimensionless angular momentum $\tilde{a}=a / r_{g}$. Noting that we can factorize our line element by a conformal factor, we get,

$$
d \sigma^{2}=\frac{r_{g}^{2}}{f^{2}(\tilde{r})}\left(1+\frac{\tilde{a}^{2}}{\tilde{r}^{2}} \cos ^{2} \theta\right)\left[\frac{f^{2}(\tilde{r}) \tilde{r}^{2}}{\Delta(\tilde{r})} d \tilde{r}^{2}+f^{2}(\tilde{r}) \tilde{r}^{2} d \theta^{2}\right],
$$

removing the $\theta$ dependence of the line element in bracket. We have, $\Delta(\tilde{r})=\tilde{r}^{2}+\tilde{a}^{2}-2 \tilde{r}$ and $f \in \mathcal{C}^{2}\left(\left[\tilde{r}_{\mathcal{H}},+\infty\left[, \mathbb{R}_{+}^{*}\right) . \quad f\right.\right.$ is a function that does not cancel in order to 
avoid singularity in conformal metrics. We are interested in the part of this submanifold outside of the horizon, so we define the $\tilde{r}$ coordinate on $\left[\tilde{r}_{\mathcal{H}}, \infty[\right.$, with

$\tilde{r}_{\mathcal{H}}=\left(1+\sqrt{1-\tilde{a}^{2}}\right)$. In order to reduce the notation, we shall skip the $\sim$ sign below in the text.

The way we wrote the line elements in Eq.(3) suggests a possible conformal transformation. Let call $\mathcal{P}_{f}$ a $2 \mathrm{D}$-manifold associated to the metrics $\mathrm{g}_{f}$ defined by,

$$
\mathbf{g}_{f}=\frac{f^{2}(\tilde{r}) \tilde{r}^{2}}{\Delta(\tilde{r})} d \tilde{r} \otimes d \tilde{r}+f^{2}(\tilde{r}) \tilde{r}^{2} d \theta \otimes d \theta .
$$

Then the application, which transforms a point $M \in \mathcal{P}$ of coordinates $\tilde{r}, \theta$ to a point $M_{f} \in \mathcal{P}_{f}$ of coordinates $\tilde{r}, \theta$ is a conformal transformation. Thus, $\mathbf{g}_{f}$ is a conformal metric and $\mathcal{P}_{f}$ a conformal manifold.

The conformal manifolds get the interesting properties that the intersections of the curves have the same angle on $\mathcal{P}$ or on $\mathcal{P}_{f}$. Indeed, the angles between the parametric lines of type $\lambda \longrightarrow(r(\lambda), \theta(\lambda))$ are the same for the poloidal manifold $\mathcal{P}$ or for the conformal manifold $\mathcal{P}_{f}$.

The expression of the conformal metrics allows us to build a conformal representation of the lines. First, removing the latitude dependence in the line elements ensures that the conformal manifold can be embedded in $\mathbb{R}^{3}$. We shall represent this embedding for $f(r)=1$. Secondly, it is known that any two-dimensional manifold is conformally flat, so we choose $f=f_{0}$ such that $\mathcal{P}_{f_{0}}$ is a flat manifold. It allows us to build a change of coordinate $R(r)$ such that we can interpret $R, \theta$ as polar coordinates on $\mathcal{P}_{f_{0}}$. By expressing the link between the conformal polar coordinates $(R, \theta)$ and the Boyer-Lindquist coordinates $(r, \theta)$, we found a way to represent in a conformal way on $\mathbb{R}_{+} \times \mathbb{R}-\mathcal{D}\left(R_{\mathcal{H}}(a)\right)$ the field lines and the curves on the poloidal manifold.

\section{Embedding of conformal poloidal manifolds}

Let us try to select an embedding of $\mathcal{P}_{f}$ in $\mathbb{R}^{3}$. Introduce the following embedding in $\mathbb{R}^{3}$,

$$
\begin{array}{ccc}
M:\left[r_{\mathcal{H}}, \infty[\times[0, \pi]\right. & \longrightarrow & \mathbb{R}^{3} \\
(r, \theta) & \longrightarrow & r f(r) \mathbf{u}(\theta)+z_{f}(r) \mathbf{e}_{z}
\end{array},
$$

where $z_{f} \in \mathcal{C}^{\infty}(\mathbb{R})$ and $\mathbf{u}(\theta)=\cos \theta \mathbf{e}_{x}+\sin \theta \mathbf{e}_{y}$ with $\left(\mathbf{e}_{x}, \mathbf{e}_{y}, \mathbf{e}_{z}\right)$ the canonical basis of $\mathbb{R}^{3}$.

Using $\mathcal{U}=\left[r_{\mathcal{H}}, \infty\left[\times[0, \pi]\right.\right.$, we interpret this embedding as the one of $\mathcal{P}_{f}$ in $\mathbb{R}^{3}$, only if the first fundamental form associated to this immersion is equal to the conformal metrics $\mathbf{g}_{f}$. This imposes a requirement on the function $z_{f}$,

$$
\left(\frac{d z_{f}}{d r}\right)^{2}=r^{2} f^{2}(r)\left[\frac{1}{\Delta(r)}-\left(\frac{d \ln (r f(r))}{d r}\right)^{2}\right] \text {. }
$$


The positiveness requirement of the right hand side brings a constraint on the choice of the function $f$ in order to obtain such embedding of $\mathcal{P}_{f}$,

$$
\left(\frac{d \ln (r f(r))}{d r}\right)^{2} \leq \frac{1}{\Delta(r)}
$$

We integrate this inequality to explicitly express the requirement on $f$. Let us choose $f(r)=1$, which obeys to the requirement of the Eq.(7). Thus we get,

$$
z_{1}(r)=z_{1, \mathcal{H}}(a)+\int_{r_{\mathcal{H}}}^{r} \sqrt{\frac{2 x-a^{2}}{\left(x-r_{\mathcal{H}}\right)\left(x+r_{\mathcal{H}}-2\right)}} d x
$$
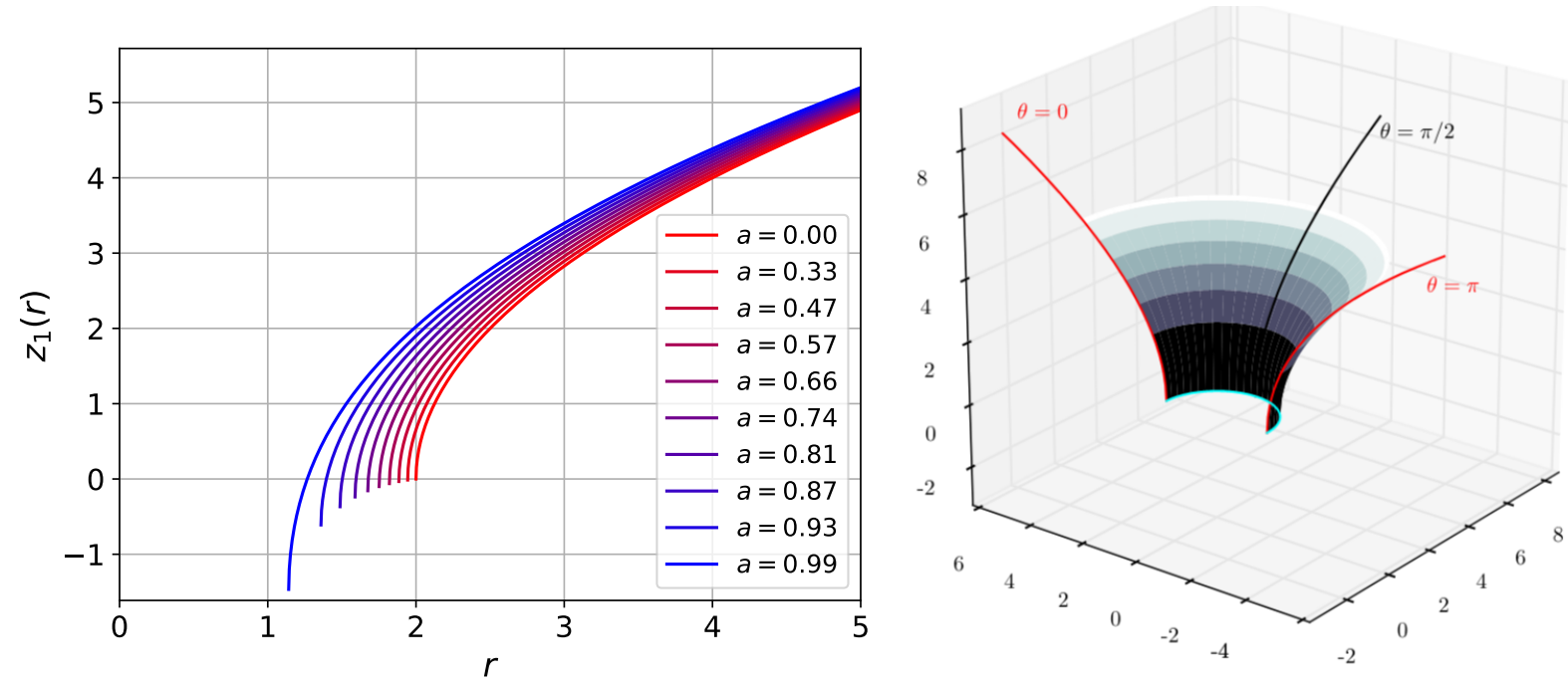

Figure 1. On the left, evolution of $z(r)$ for different values of $a$. The curves obtained for $a=1$ reach $-\infty$ where $r$ reaches $r_{\mathcal{H}}$.

Figure 2. Plot of the poloidal submanifold between $\theta=0$ and $\theta=\pi$. On the right, the embedding of the conformal poloidal manifold $\mathcal{P}_{1}$ for $a=0.9$. The red solid line is the set of points with $\theta=0$ and $\theta=\pi$, which allows us to interpret it as the $O_{z}$ axis.

For all $a$ with $|a|<1$ this integral is correctly defined on $] r_{\mathcal{H}},+\infty[$, because,

$$
\sqrt{\frac{2 x-a^{2}}{\left(x-r_{\mathcal{H}}\right)\left(x+r_{\mathcal{H}}-2\right)}} \underset{x \rightarrow r_{\mathcal{H}}}{\sim} \frac{r_{\mathcal{H}}}{\sqrt{2\left(r_{\mathcal{H}}-1\right)}} \frac{1}{\sqrt{x-r_{\mathcal{H}}}},
$$

which can be integrated on $\left.] r_{\mathcal{H}}, r\right]$. We choose $z_{1, \mathcal{H}}(a)$ equal to

$$
z_{1, \mathcal{H}}(a)=\lim _{r \rightarrow+\infty} \int_{r_{\mathcal{H}}}^{r} \sqrt{\frac{2 x-a^{2}}{x^{2}+a^{2}-2 x}} d x-2 \sqrt{2} \sqrt{r-2},
$$

in order to obtain the same behavior at infinity for the different functions, $z_{1}$ when we change the value of $a$. The curves $z_{1}$ are plotted in Fig.(3) and the corresponding embedding surface on Fig.(2). According to the previous equivalence, for all $a$ with $|a|<1$ we have $z_{1} \underset{r \rightarrow r_{\mathcal{H}}}{\sim} \sqrt{r-r_{\mathcal{H}}}$. For $|a|=1$ we need to have $z_{1} \underset{r \rightarrow r_{\mathcal{H}}}{\sim} \ln r-r_{\mathcal{H}}$. We note $\mathcal{P}_{1}$ the sub-manifold embedded in $\mathbb{R}^{3}$ presented in this section. 


\section{Conformally flat poloidal manifold}

All bidimensional manifolds are locally conformally flat. We are therefore looking for the function $f_{0}$ and the radial coordinates $R(r)=r f_{0}(r)$ such that the conform metrics in Eq.(3) is the one of a flat manifold in polar coordinates. Such condition requires the cancellation of Eq.(6), which can be rewritten with the both conditions,

$$
\begin{aligned}
\frac{d \ln R}{d r} & =\frac{1}{\sqrt{\Delta(r)}} \\
f_{0}(r) & =R / r .
\end{aligned}
$$

Then we get,

$$
R(r)=R_{\mathcal{H}} \exp \int_{r_{\mathcal{H}}}^{r} \frac{d x}{\sqrt{x^{2}+a^{2}-2 x}}=\frac{1}{2}\left(r-1+\sqrt{r^{2}-2 r+a^{2}}\right),
$$

where $R_{\mathcal{H}}=R\left(r_{\mathcal{H}}\right)$. As in Eq.(3), we can easily show that this integral is properly defined.

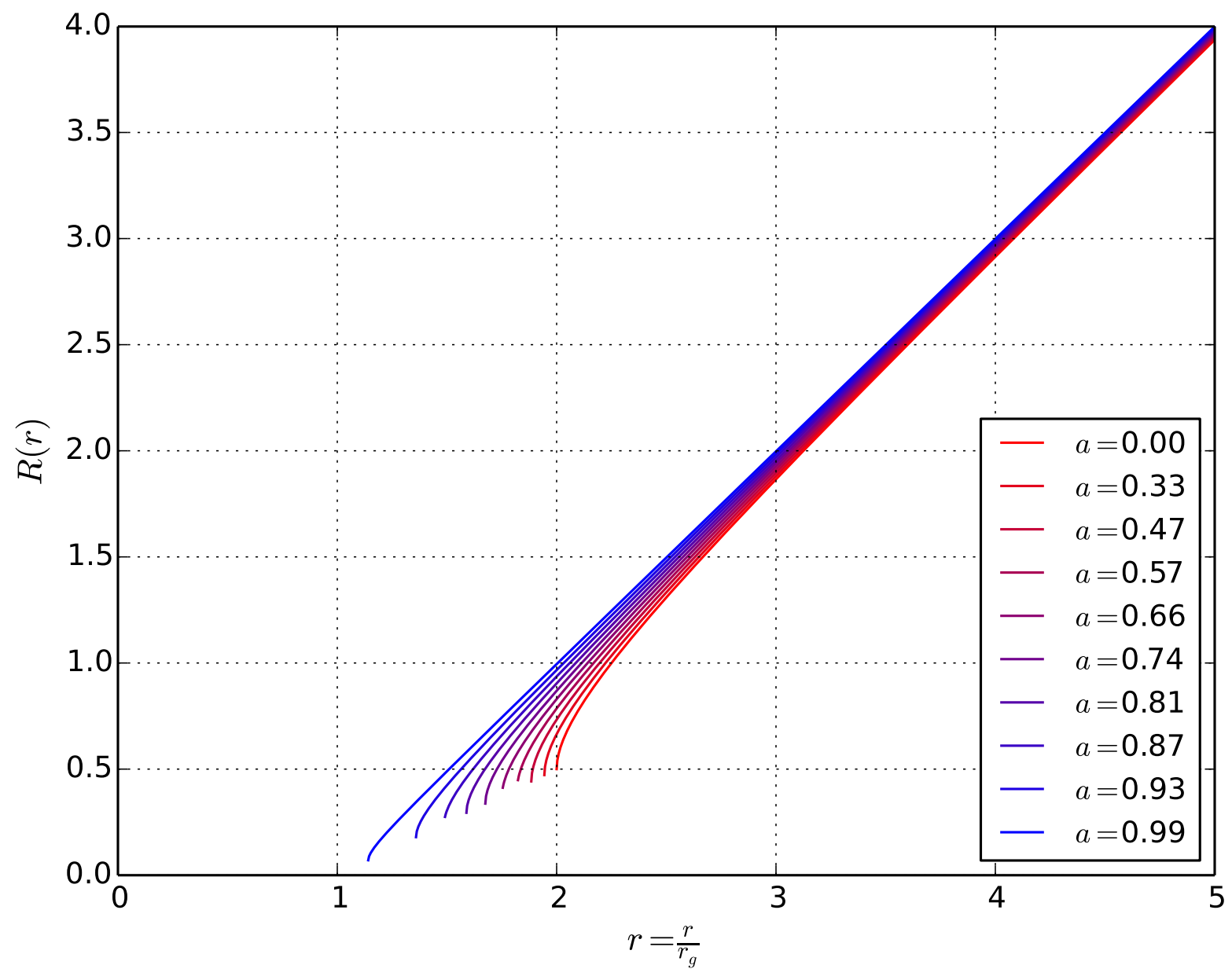

Figure 3. Evolution of $R(r)$ for different values of $a$. 
We have set $R_{\mathcal{H}}=R\left(r_{\mathcal{H}}\right)=\sqrt{1-a^{2}} / 2$, in order to have $R \underset{r \rightarrow \infty}{\sim} r$. This coordinate is the so-called quasi-isotropic coordinate.

The expression of Eq.(11) calculated for $a^{2}<1$ can be extended, by continuity, to extreme Kerr black holes for which $a^{2}=1$. The expression, Eq.(11), can also be inverted in order to express $r$ as a function of $R(r)$,

$$
r=1+R(r)+\frac{1-a^{2}}{4 R(r)}=R(r)\left(1+\frac{1+a}{2 R(r)}\right)\left(1+\frac{1-a}{2 R(r)}\right) .
$$

Then the curves $\lambda \longrightarrow(r(\lambda), \theta(\lambda))$ representing the following Cartesian coordinates,

$$
\begin{aligned}
& \varpi(r, \theta)=R(r) \sin \theta \\
& Z(r, \theta)=R(r) \cos \theta,
\end{aligned}
$$

cross themselves with correct apparent angles. We will call these coordinates the quasiisotropic Cartesian coordinates. In the following we used $\mathcal{P}_{0}$ for the conformal manifold $\mathcal{P}_{f_{0}}$.

\section{Representation of two orthogonal families of curve}

To illustrate the relevance of the conformal embedding or quasi-isotropic coordinates previously introduced, let us introduce two families of curves which are chosen in order to map the manifold and to be orthogonal between themselves. The first families $\mathcal{L}_{\varpi}$ is,

$$
\begin{aligned}
& r_{\varpi}(\lambda)=\frac{\varpi}{\sin \lambda} \\
& \theta_{\varpi}(\lambda)=\lambda .
\end{aligned}
$$

This family covers $\mathcal{P}$. We define the second family $\mathcal{L}_{Z}: M\left(r_{Z}(\lambda), \theta_{Z}(\lambda)\right)$ as the curves that cross perpendicurlarly the curves of the first family. In order to satisfy this request the speed vector of the second family $\mathbf{v}_{Z}=\dot{r}_{Z} \boldsymbol{\partial}_{r}+\dot{\theta}_{Z} \boldsymbol{\partial}_{\theta}$ (indexed by $Z$ ) needs to be chosen orthogonal to the speed vector of the first family. Thus, the function $\left(r_{Z}(\lambda), \theta_{Z}(\lambda)\right)$ of the second family $\mathcal{L}_{Z}$ must satisfy the following differential system,

$$
\begin{aligned}
& \dot{r}_{Z}(\lambda)= \pm \frac{1}{h_{r}^{2}\left(r_{Z}, \theta_{Z}\right)} \dot{\theta_{\varpi}} \\
& \dot{\theta}_{Z}(\lambda)=\mp \frac{1}{h_{\theta}^{2}\left(r_{Z}, \theta_{Z}\right)} \dot{r}_{\varpi} .
\end{aligned}
$$

We stop the integration of this equation where the line reaches the boundary of the manifold or reaches a limiting radius. On Fig.(4) we represented these families for different kind of representation. First of all we directly used Cartesian coordinates attached to Boyer-Lindquist coordinates $(x=r \sin \theta, y=r \cos \theta)$, which are not a conformal representation. Then we used the surface embedded into $\mathbb{R}^{3}$ presented in Fig.(2). And finally we used the Cartesian coordinates attached to quasi-isotropic coordinates.

As expected, the intersection between two curves of the two orthogonal families appears orthogonal, in a conformal representation, either on the embedded surface or using quasi-isotropic coordinates). The intersection does not appear orthogonal when we used Boyer-Lindquist's coordinates. 

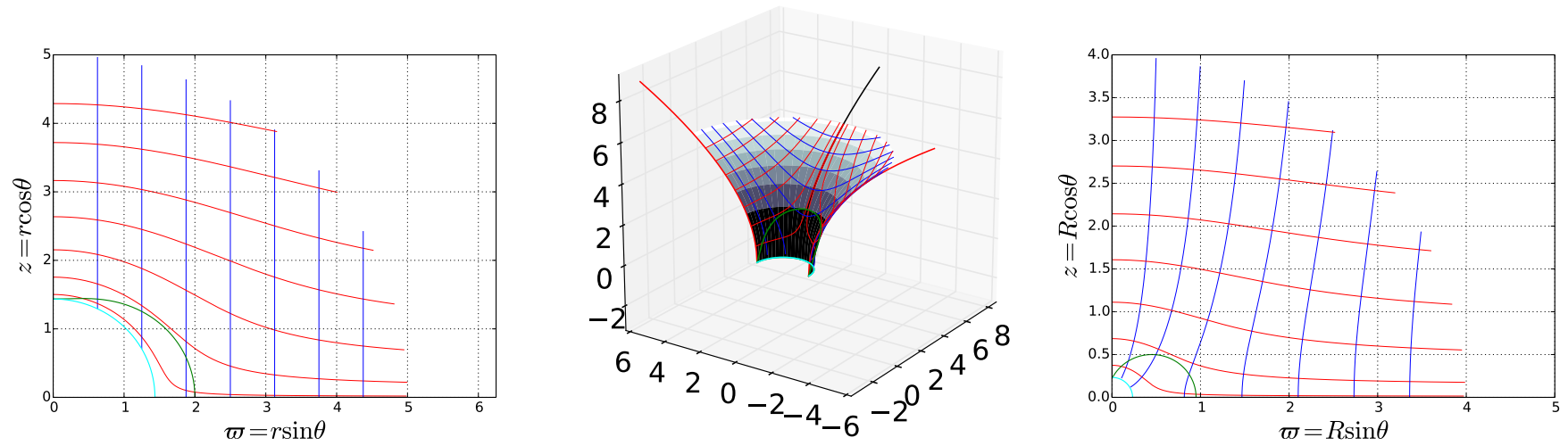

Figure 4. Two subsets of the orthogonal families of curve $\mathcal{L}^{1}$ (blue) and $\mathcal{L}^{2}$ (red) represented in the Boyer-Lindquist Cartesian coordinates (left), in the embedded manifold (center), in the conformal Cartesian coordinates (right). The chosen spin is $a=0.9$. We also plotted the ergosphere in green. The horizon is represented in cyan. The $O_{z}$ and $O_{\varpi}$ axis are drawn in black solid lines.

\section{Boyer-Lindquist and conformal representations of field lines}

To insist on the importance to represent magnetic field lines with a conformal set of coordinates, let us give the different representations of some examples of typical magnetic field configurations. Remind the classical result in the axi-symmetric assumption that the poloidal magnetic field is entirely determined by the value of the magnetic flux $A$ which crosses the circle of coordinates $(r, \theta)$,

$$
\mathbf{B}_{p}=\frac{\nabla A \times \boldsymbol{\epsilon}_{\phi}}{h_{\phi}} .
$$

This equation implies that the magnetic field $B^{\hat{\theta}}$ must be 0 on the horizon because $h_{r} \underset{r \rightarrow r_{\mathcal{H}}}{\rightarrow} \infty$. More details are given in Chantry et al. [2018]. This result implies that the poloidal magnetic field line corresponds to the isocontours of the magnetic flux $A=$ Cst. Let us give some examples of typically used magnetic-flux fields and represent their isovalues in the Boyer-Lindquist coordinates or in the quasi-isotropic coordinates.

\subsection{Intersection of curves and event horizon}

It should also be noted that the curve which crosses the horizon enters inside it perpendicularly (except for some specific cases). This implies that most of magnetic field lines are always perpendicular to the event horizon surface, except for specific cases. Indeed for a curve $(r(\theta), \theta)$, such that $r\left(\theta_{0}\right)=r_{\mathcal{H}}$, the angle $\alpha_{\mathcal{H}}$ between the curve and $\boldsymbol{\partial}_{r}$ at the horizon verifies the equation,

$$
\cos \alpha_{\mathcal{H}}=\lim _{\theta \rightarrow \theta_{0}} \frac{\frac{d r}{d \theta}}{\sqrt{r^{2}-2 r+a^{2}+\left(\frac{d r}{d \theta}\right)^{2}}} .
$$


The angle $\alpha_{\mathcal{H}}$ corresponds to the intrinsic angle. The apparent angles correspond everywhere to the intrinsic angles if we use a conformal representation. If we do not have $d r / d \theta \rightarrow 0$ on the horizon, we get $\alpha_{\mathcal{H}}=0[\pi]$. The curve which defines the ergosphere $r_{\mathcal{E}}(\theta)=r_{g}\left(1+\sqrt{1-a^{2} \cos ^{2} \theta}\right)$ fulfills the condition $d r / d \theta \rightarrow 0$ when this curve touches the horizon at its pole. This is why on the pole of the horizon the ergosphere touches it with this non-orthogonal angles $\alpha_{\mathcal{H}, \mathcal{E}}$ on the conformal representation in Fig.(4). In fact a simple calculation gives

$$
\cos \alpha_{\mathcal{H}, \mathcal{E}}=a .
$$

For a curve of constant magnetic flux $A$, the local value of $d r / d \theta$ is given by $-\partial_{\theta} A / \partial_{r} A$. Then if $-\partial_{\theta} A / \partial_{r} A$ does not reach 0 on the horizon, every poloidal field line, which reaches the horizon touches it perpendicularly.

In the representation of GSE solutions, see Nathanail and Contopoulos [2014], the curves of constant magnetic flux does not seem to enter perpendicularly in the horizon. Let us see how the field line represented on this paper look like when we use a conformal representation. Let us also check that these curves enter in the horizon perpendicularly to it. We will compare a classic representation using Boyer-Lindquist Cartesian coordinates and the quasi-isotropic coordinates.

\subsection{Vertical field lines}

Let us start with a vertical magnetic field line configuration,

$$
A(r, \theta)=A_{\mathcal{H}}\left(\frac{r \sin \theta}{r_{\mathcal{H}}}\right)^{2} .
$$
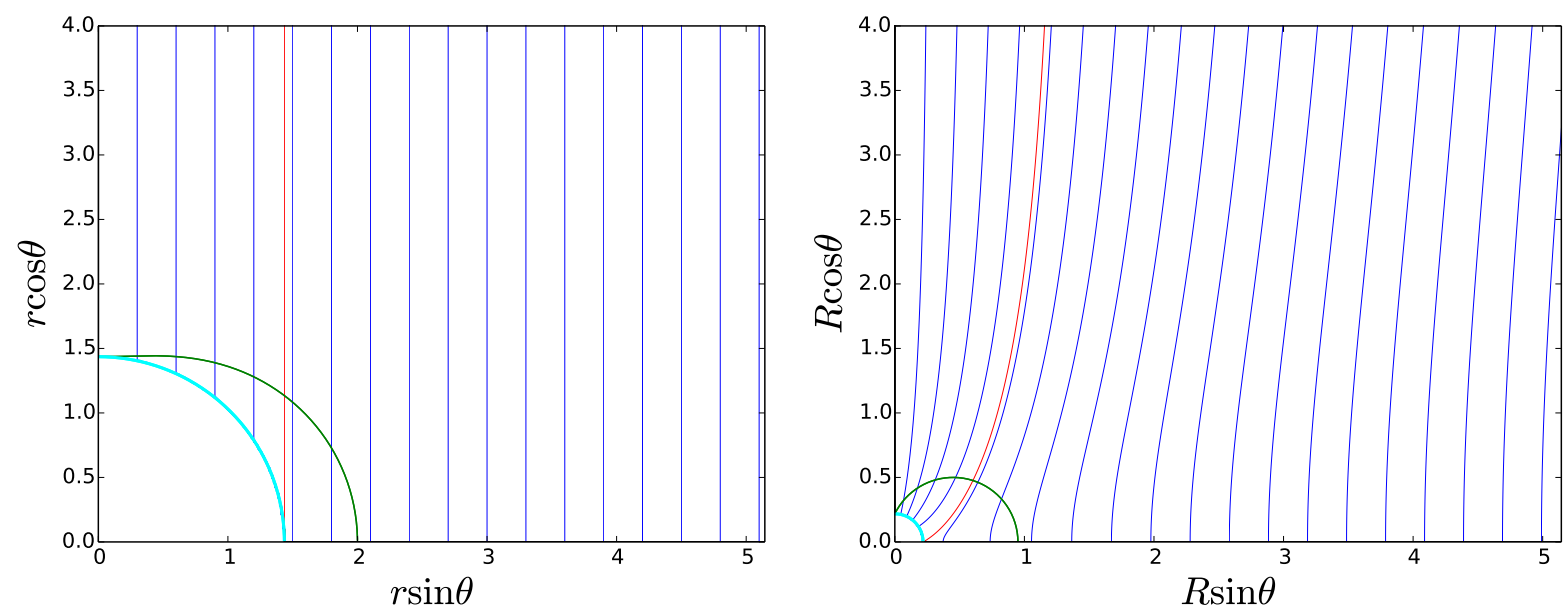

Figure 5. Representation of a vertical field line, in the Boyer-Lindquist Cartesian coordinates (left), in the quasi-isotropic Cartesian coordinates. We choose $a=0.9$, the horizon is represented in cyan, the ergosphere in green and the axis in black.

For this configuration $\partial_{\theta} A / \partial_{r} A$ reaches 0 only for $\theta=\pi / 2$. Then the poloidal field line curve reaches the black hole horizon perpendicularly to it except the one which 
touches the horizon on the equator. This property appears correctly on a conformal representation. We plotted the only line which does not cross perpendicularly in red on Fig.(5). A calculation leads to $\cos \alpha_{\mathcal{H}}=r_{\mathcal{H}} / \sqrt{3 r_{\mathcal{H}}-2 a^{2}}$.

\subsection{Paraboloidal field lines}

In Fig.(6), we plot a paraboloidal configuration of magnetic field lines,

$$
A(r, \theta)=A_{\mathcal{H}}\left(\frac{r+r_{0}}{r_{\mathcal{H}}+r_{0}}\right)^{\nu}(1-|\cos \theta|) .
$$
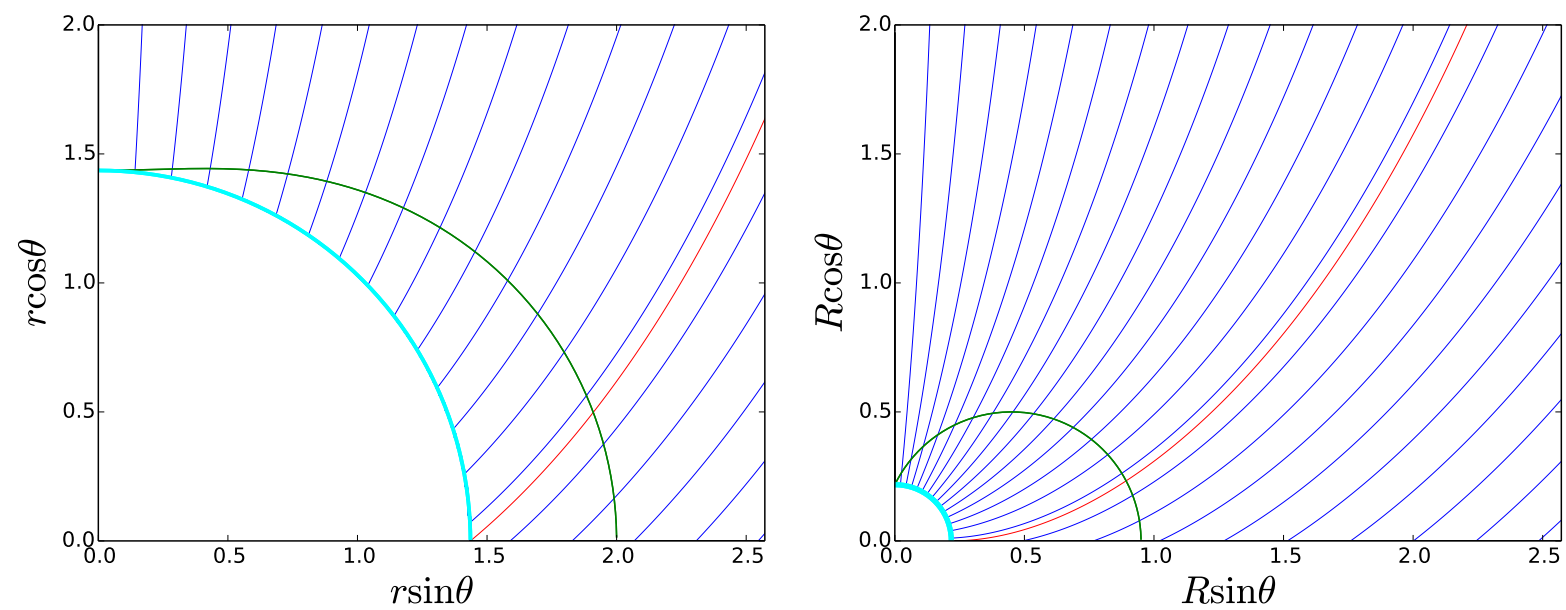

Figure 6. Representation of paraboloidal field lines for $\nu=2$, in the Boyer-Lindquist Cartesian coordinates (left), in the quasi-isotropic Cartesian coordinates (right). We choose $a=0.9$, the horizon is represented in cyan, the ergosphere in green and the axis in black.

For this field $\partial_{\theta} A / \partial_{r} A$ does not reach 0 on the horizon, then the field line enters in it perpendicularly to it. We observe again that the angles of intersection between the field lines and the horizon appear correctly on the conformal representation.

\subsection{Field lines with a magnetosphere}

Then in Fig.(7), we plot a configuration of magnetic field lines with a magnetosphere (closed field lines),

$$
A(r, \theta)=A_{\mathcal{H}} g(r) \sin ^{2} \theta,
$$

where $g$ is a continuous function, which decreases between $r_{\mathcal{H}}$ and $r_{M}$ (magnetosphere radius) and increases for larger radii. In Fig. 7, we have chosen the following form for $g(r)$,

$$
g(r)=2-\tanh \left(r-r_{\mathcal{H}}\right)-\frac{\exp \left(-0.01\left(r-3.5 r_{\mathcal{H}}\right)^{2}\right)}{2} .
$$



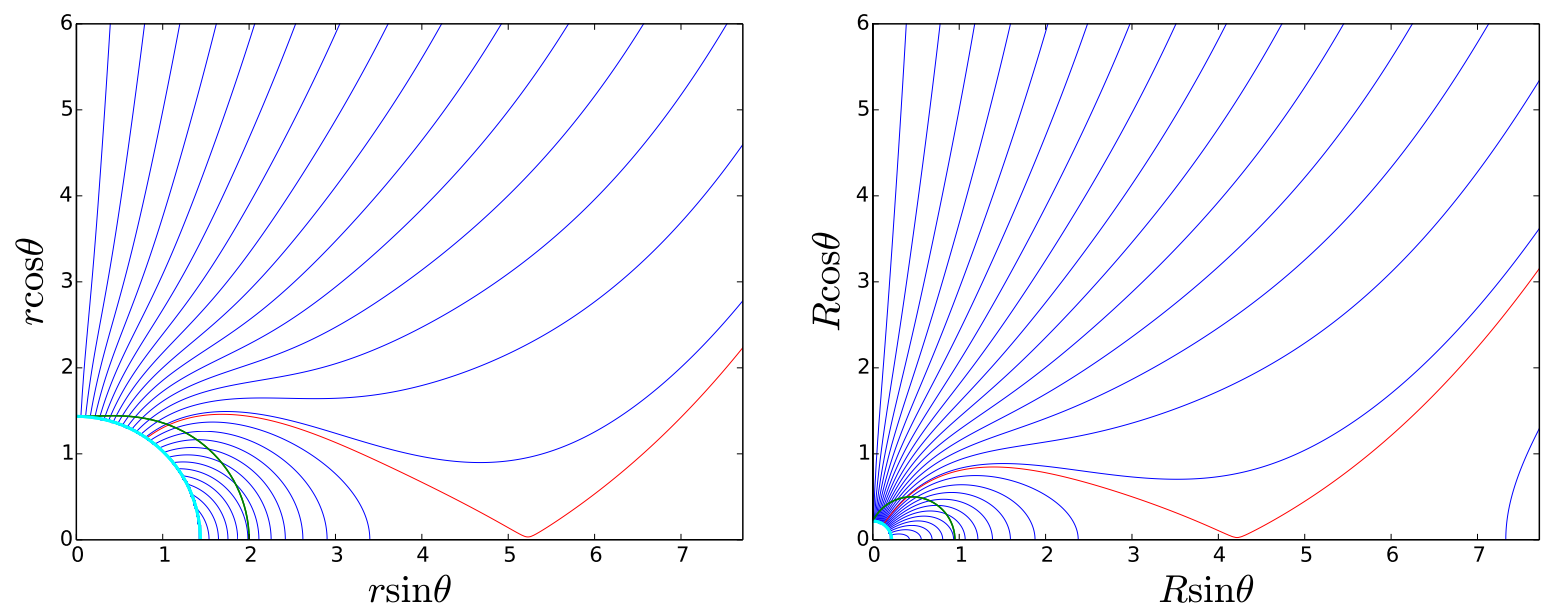

Figure 7. Plot of the magnetic field lines with a magnetosphere, in the BoyerLindquist Cartesian coordinates (left) and in the quasi-isotropic Cartesian coordinates (right). We choose $a=0.9$ and $g(r)$ is given in the main text. The horizon is represented in cyan, the ergosphere in green and the axis in black.

In Fig.(7), we plot in red the limiting line between the open field line and the magneto-sphere. Because $d g / d r \neq \infty$ on the horizon, everywhere on the horizon the field lines enter in it perpendicularly, which is correctly plotted on the quasi-isotropic Cartesian coordinates representation.

\section{Conformal factor}

Concerning the proper representation of angles, the use of quasi-isotropic Cartesian coordinates is more practical than the representation on the surface embedded in $\mathbb{R}^{3}$, since the representation using these coordinates avoids the use of 3D representation. Nevertheless, the representation of distances is better for the embedded representation. Indeed, on a conformal representation the intrinsic length of an infinitesimal segment correspond to the apparent length multiplied by the conformal factor at this point. The closer to one is $\Omega$, the better is the representation of distance. Remember that for the conformally flat manifold, the conformal factor is,

$$
\Omega_{f_{0}, a}^{2}(r, \theta)=\frac{r^{2}+a^{2} \cos ^{2} \theta}{R_{a}^{2}},
$$

which is between 1 and $8\left(1+\sqrt{1-a^{2}}\right) /\left(1-a^{2}\right)$. Therefore for the embedded surfaces plotted in Fig.(2), we get,

$$
\Omega_{1, a}^{2}(r, \theta)=\frac{r^{2}+a^{2} \cos ^{2} \theta}{r^{2}}
$$

which is between 1 and 2. The values of the conformal factor are plotted in Fig.(8).

We observe that the conformal factor of the conformally flat manifold $\mathcal{P}_{0}$ becomes quite large near the horizon specially when $a$ is close to 1 . It means that we have a bad 

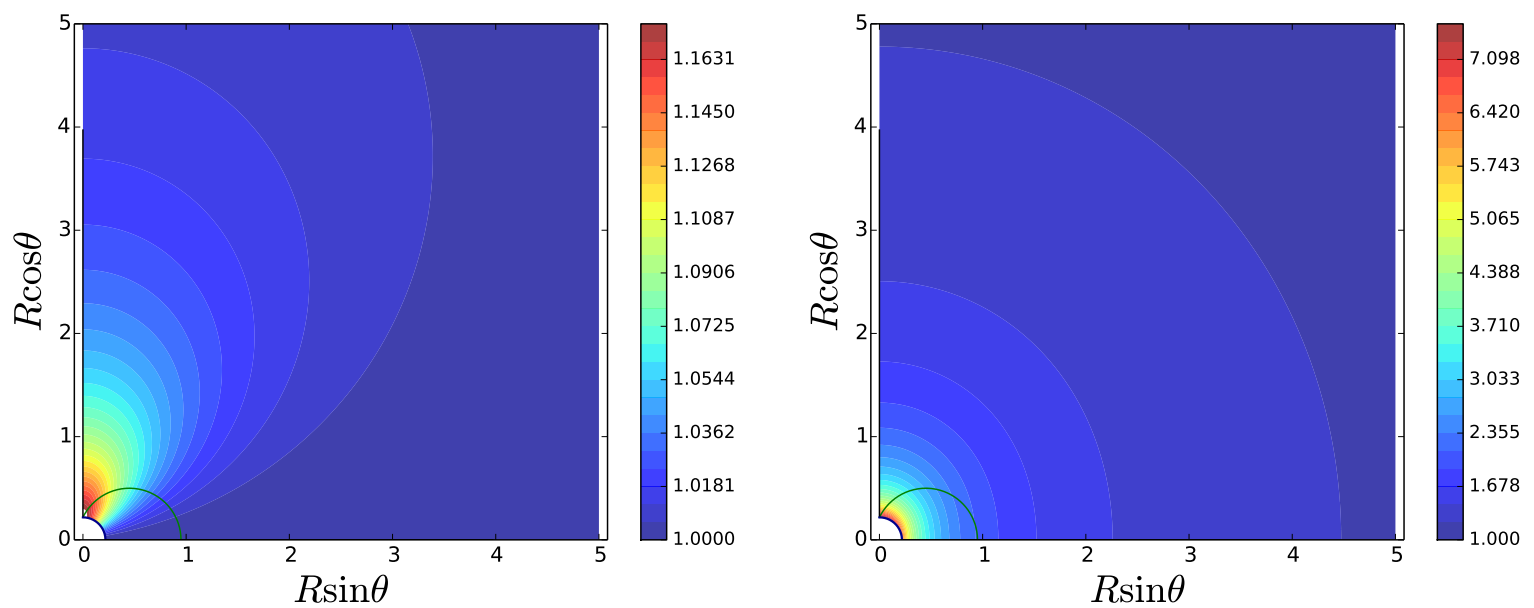

Figure 8. Conformal factor in quasi-isotropic Cartesian coordinates for the embedded conformal manifold in $\mathbb{R}^{3}$ named $\mathcal{P}_{1}$ (left) and for the conformally flat manifold $\mathcal{P}_{0}$ (right). The spin is chosen to the value of $a=0.9$.

representation of distances near the horizon. This is not the case for $\mathcal{P}_{1}$, for which the conformal factor is limited by $\sqrt{2}$ and we have a quite good representation of distances close to the equator $\Omega_{1, a}^{2}(r, \pi / 2)=1$.

\section{Discussion}

In this work we present a simple way to get a planar and conformal representation of fields and curves on the Kerr poloidal sub-manifold. This representation is based on a construction of a conformal radial coordinates as functions of the Boyer-Lindquist radial coordinates Eq.(11). This system can be inverted in Eq.(12). In this representation, the size of the horizon decreases with the spin of the black hole and disappears for extremely rotating Kerr Black Hole. We also construct the quasi-isotropic Cartesian coordinate in Eq.(14).

In stationary MHD, except for specific case the poloidal magnetic field line enters in the horizon perpendicularly to it. Then it is important to have a conformal representation in order to check quickly this property of these solutions on our representation.

We also obtain a conformal representation based on an isometric embedding in $\mathbb{R}^{3}$ of a conformal manifold. This representation is not a planar one, but the conformal factor is globally smaller than the one for a planar representation. For this reason, we get a better representation of distances compared to the planar representation where the maximum value of the conformal factor is infinity for $a \longrightarrow 1$, when the conformal factor is between 1 and $\sqrt{2}$.

The only way to obtain a satisfying representation of distances is to perform an isometric embedding. In the future we may use the algorithm given in Hotz and Hagen [2004] or another one method, to numerically compute an isometric embedding in $\mathbb{R}^{3}$ of 
poloidal sub-manifold. This embedding could give to us better intuition concerning the motion in the poloidal sub-manifold.

\section{Acknowledgments}

L. Chantry thanks Ioannis Contopoulos for bringing to his attention on this subject. C. Sauty thanks LUPM and IN2P3 for hosting him during his CNRS delegation. This work was supported by the Programme National des Hautes Energies of CNRS/INSU with INP and IN2P3, co-funded by CEA and CNES, and the Action Fédératrice CTA from the Observatoire de Paris.

\section{References}

Mihai Bondarescu, Miguel Alcubierre, and Edward Seidel. Isometric embeddings of black-hole horizons in three-dimensional flat space. Classical and Quantum Gravity, 19(2):375-391, Jan 2002. doi: 10.1088/0264-9381/19/2/311.

L. Chantry, V. Cayatte, C. Sauty, N. Vlahakis, and K. Tsinganos. Nonradial and nonpolytropic astrophysical outflows. X. Relativistic MHD rotating spine jets in Kerr metric. Astronomy and Astrophysics, 612:A63, Apr 2018. doi: 10.1051/0004-6361/ 201731793.

Eric Gourgoulhon. An introduction to the theory of rotating relativistic stars. arXiv e-prints, art. arXiv:1003.5015, Mar 2010.

Yong-Jin Han and Hyun-Chul Lee. Embedded Diagrams of Kerr and Non-Kerr black Holes. arXiv e-prints, art. arXiv:1607.06535, Jul 2016.

Stanislav Hledík, Zdenĕk Stuchlík, and Alois Cipko. Visualizing Spacetimes via Embedding Diagrams. In The Eleventh Marcel Grossmann Meeting On Recent Developments in Theoretical and Experimental General Relativity, Gravitation and Relativistic Field Theories, pages 2299-2301, Sep 2008. doi: 10.1142/9789812834300_ 0392.

Ingrid Hotz and Hans Hagen. Isometric embedding for a discrete metric. In Guido Brunnett, Bernd Hamann, Heinrich Müller, and Lars Linsen, editors, Geometric Modeling for Scientific Visualization, pages 19-35, Berlin, Heidelberg, 2004. Springer Berlin Heidelberg. ISBN 978-3-662-07443-5.

Michael Jasiulek and Mikołaj Korzyński. Isometric embeddings of 2-spheres by embedding flow for applications in numerical relativity. Classical and Quantum Gravity, 29(15):155010, Aug 2012. doi: 10.1088/0264-9381/29/15/155010. 
J. F. Mahlmann, P. Cerdá-Durán, and M. A. Aloy. Numerically solving the relativistic Grad-Shafranov equation in Kerr spacetimes: numerical techniques. MNRAS, 477: 3927-3944, July 2018. doi: 10.1093/mnras/sty858.

A. Nathanail and I. Contopoulos. Black Hole Magnetospheres. APJ, 788:186, June 2014. doi: $10.1088 / 0004-637 \mathrm{X} / 788 / 2 / 186$.

Zhen Pan. Magnetosphere structure of a Kerr black hole: Marginally force-free equatorial boundary condition. Phys. Rev. D, 98(4):043023, Aug 2018. doi: 10.1103/PhysRevD.98.043023.

Shannon Ray, Warner A Miller, Paul M Alsing, and Shing-Tung Yau. Adiabatic isometric mapping algorithm for embedding 2-surfaces in euclidean 3-space. Classical and Quantum Gravity, 32(23):235012, nov 2015. doi: 10.1088/0264-9381/32/23/ 235012. URL https://doi org/10.1088\%2F0264-9381\%2F32\%2F23\%2F235012.

Larry Smarr. Surface geometry of charged rotating black holes. Phys. Rev. D., 7:289295, Jan 1973. doi: 10.1103/PhysRevD.7.289. URL https://link.aps.org/doi/ 10.1103/PhysRevD.7.289.

Wolfgang Tichy, Jonathan R McDonald, and Warner A Miller. New efficient algorithm for the isometric embedding of 2-surface metrics in three dimensional euclidean space. Classical and Quantum Gravity, 32(1):015002, dec 2014. doi: 10.1088/0264-9381/32/ 1/015002. URL https://doi.org/10.1088\%2F0264-9381\%2F32\%2F1\%2F015002. 